\section{Case Report}

Korean J Transplant 2021;35:48-52 https://doi.org/10.4285/kjt.20.0041
Received September 8, 2020

Revised January 26, 2021

Accepted February 17, 2021

Corresponding author: Phyo Wai Lwin Department of Nephrology, University of Medicine, Mandalay, Chan Aye Thar San Township, Mandalay Division, Myanmar Tel: +95-9972349611

Fax: +95-9972349611

E-mail: Iphyowai84@gmail.com

(C) The Korean Society for Transplantation This is an Open Access article distributed under the terms of the Creative Commons Attribution Non-Commercial License (http://creativecommons.org/licenses/ by-nc/4.0/) which permits unrestricted non-commercial use, distribution, and reproduction in any medium, provided the original work is properly cited.

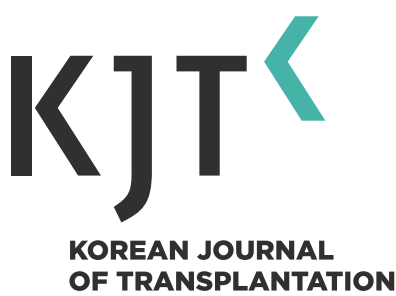

pISSN 2671-8790

eISSN 2671-8804

\title{
Posttransplantation tuberculosis management in terms of immunosuppressant cost: a case report in Myanmar
}

\author{
Phyo Wai Lwin ${ }^{1}$, Yi Yi Htun ${ }^{2}$, Aung Kyaw Myint ${ }^{3}$, Htar Kyi Swe ${ }^{1}$ \\ ${ }^{1}$ Department of Nephrology, University of Medicine, Mandalay, Mandalay, Myanmar \\ ${ }^{2}$ Department of Pharmacology, University of Medicine, Mandalay, Mandalay, Myanmar \\ ${ }^{3}$ Department of Nephrology, Mandalay General Hospital, Mandalay, Myanmar
}

\begin{abstract}
Drug interactions between anti-tuberculosis and immunosuppressive medications after renal transplantation are a common problem in Myanmar. The efficacy of both types of drugs can be reduced during the treatment period, which can lead to graft failure and flare-ups of infection. Drug adjustments, with frequent monitoring and close follow-up, are crucial in this period. Ketoconazole decreases tacrolimus metabolism by inhibiting cytochrome P450-3A5 enzymes and P-glycoprotein. It is cost effective and has been frequently used to reduce the dose and cost of tacrolimus. Here, we report the case of a 56-year-old male renal transplant recipient with anti-tuberculosis medications.
\end{abstract}

Keywords: Post renal transplant tuberculosis; Immunosuppressant cost; Drug Interaction

\section{INTRODUCTION}

Tuberculosis (TB) is caused by pathogenic species of the Mycobacterium tuberculosis complex. Individuals with impaired immune responses, such as solid organ transplant (SOT) recipients, have a higher likelihood of developing TB than immunocompetent persons $[1,2]$. TB is a common infection associated with considerable morbidity and mortality. Its clinical presentation in immunocompromised individuals, including transplant recipients, is often atypical and diverse. This leads to delayed diagnosis and advanced disease at the time of diagnosis. The higher toxicity of treatment and the concurrent use of immunosuppressive medications with drug interactions further increase the complexity of management. Because of various interactions between anti-TB medications and the drugs used in renal transplant patients, fluctuations in immunosuppressant levels and reduction in the efficacy of anti-TB medications can occur [3]. Rifampicin-containing anti-TB regimens can decrease tacrolimus levels, whereas ketoconazole treatment reduces the efficacy of anti-TB drugs. These drug interactions can result in graft failure and anti-TB drug failure in transplant recipients taking anti-TB medications. Tacrolimus remains the backbone of modern immunosuppressive therapy for SOT recipients. Owing to its adverse effects, narrow safety margin, and large variability in absorption and metabolism, clinical monitoring of tacrolimus exposure is necessary [4-6]. Tacrolimus is the most important immunosuppressive drug for kidney transplant patients. It is mainly metabolized by cytochrome P450-3A5 (CYP3A5) enzymes and P-glycoprotein in the liver and intestine [1-3]. Ketoconazole can inhibit CYP3A5 enzymes and P-glycoprotein, and thereby can decrease the metabolism of tacrolimus. As ketoconazole is an inexpensive drug, it has been frequently used to reduce the dose and cost of tacrolimus. A few studies have demonstrated 


\section{HIGHLIGHTS}

- Transplantation has been associated with various infections owing to the use of immunosuppressive medications after the procedure. Tuberculosis is one of the major health problems in developing tropical countries.

- Anti-tuberculosis medications have well-known adverse effects and many drug interactions with immunosuppressant drugs.

- Important interactions between anti-tuberculosis drugs and tacrolimus, which lower the immunosuppressant levels and reduce the potency of anti-tuberculosis drugs.

the financial benefits and short-term safety of such a practice in kidney transplant patients $[4,5]$. The addition of ketoconazole has been reported to considerably reduce the tacrolimus dose (by 58.7\%) and the medical cost (by $56.9 \%$ ) at 6 months [7]. A previous study has shown that 2 years after kidney transplantation, patients treated with ketoconazole achieved benefits both in terms of tacrolimus dose reduction and financial savings. Coadministration of ketoconazole was not associated with a higher risk of rejection or inferior graft survival [8].

\section{CASE REPORT}

The patient provided informed consent for the publication of this report to Hospital ethical board of Mandalay General Hospital and board allowed for publication.

Our patient was a 56-year-old man who lives in the forest region of Myanmar. He underwent renal transplantation in India for end-stage kidney failure due to hypertension in July 2018. The induction therapy used was an anti-thymocyte globulin-based regimen. He regularly took tacrolimus, mycophenolate mofetil, and steroids after the operation. He was well in the early posttransplantation period. One month after the operation, his creatinine level was $1.5 \mathrm{mg} / \mathrm{dL}$ and his serum tacrolimus level was within the target range according to the protocol. However, he was admitted to the hospital for palpitations and breathlessness persisting for 2 days on September 17, 2019, 2 months after the kidney transplantation. At that time, his condition was severe and he was unable to lie flat. He denied having fever, cough, night sweats, loss of appetite, and chest pain.

On physical examination, his blood pressure was $130 / 80 \mathrm{mmHg}$. However, his pulse rate was 152 beats/ min, and electrocardiography showed atrial fibrillation. Coarse crepitations in both lung fields were also observed. Enlargement of cervical and axillary lymph nodes was not observed. However, he had leg edema. The blood tests showed normal results, except for low hemoglobin value, low leukocyte counts, and elevated C-reactive protein and blood sugar levels. Therefore, we treated the patient for acute heart failure with chest infection, proximal atrial fibrillation, and anemia of unknown cause.

We administered amiodarone for atrial fibrillation and insulin for sugar control. Antibiotics were administered for chest infection because of the high $\mathrm{C}$-reactive protein level. Chest radiography (posteroanterior view) suggested a bronchogenic carcinoma (right middle lobe) (Fig. 1). We performed computed tomography (CT) for further assessment and management. Chest CT also suggested a bronchogenic carcinoma (right upper lobe, lower anterior segment) and a spiculated mass lesion. Therefore, we consulted a chest physician for further management (Fig. 2).

The chest physician performed bronchoscopy, and the bronchial lavage test revealed sputum-positive pulmonary TB (acid-fast bacilli [AFB] were seen in the bronchial lavage examination). Therefore, an anti-TB regimen (isoniazid, rifampicin, ethambutol, and pyrazinamide) was initiated after consulting with the chest physician. Before anti-TB treatment, his tacrolimus level was $12.99 \mathrm{ng} / \mathrm{mL}$ with a

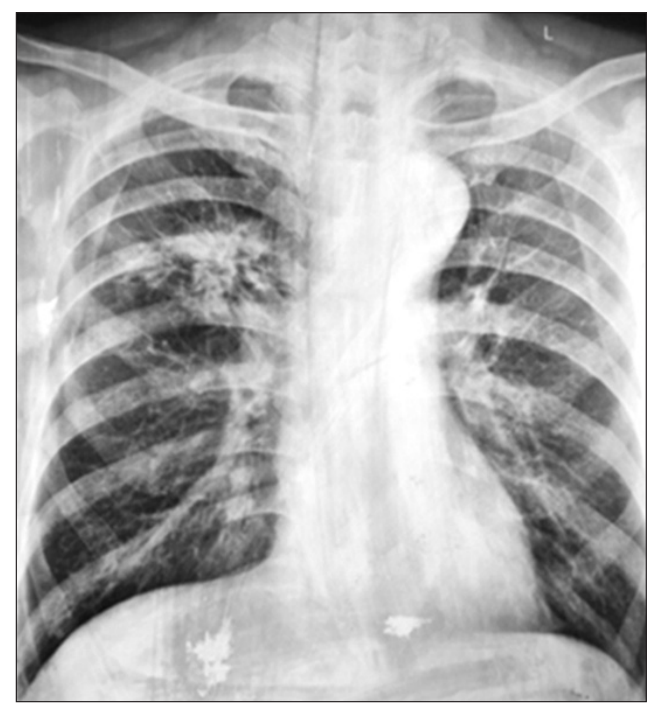

Fig. 1. Chest radiograph of the patient. 

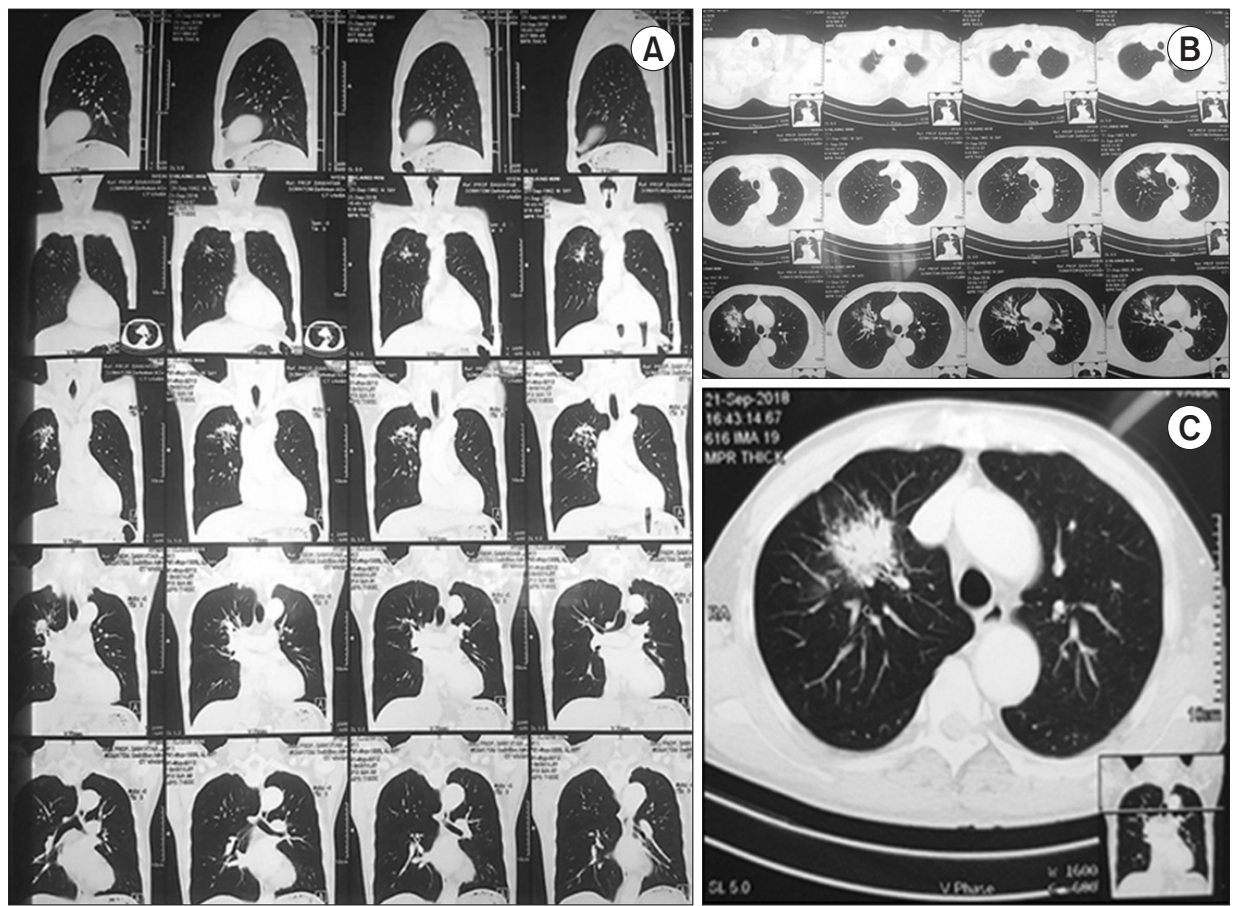

Fig. 2. Computed tomography scan of the patient. (A) Speculated mass lesion in right lower segments and air space opacity with internal air bronchogram. (B) Consolidation in anterior segment of right upper lobe. (C) Soft tissue lesion in right lung field with speculated margin.

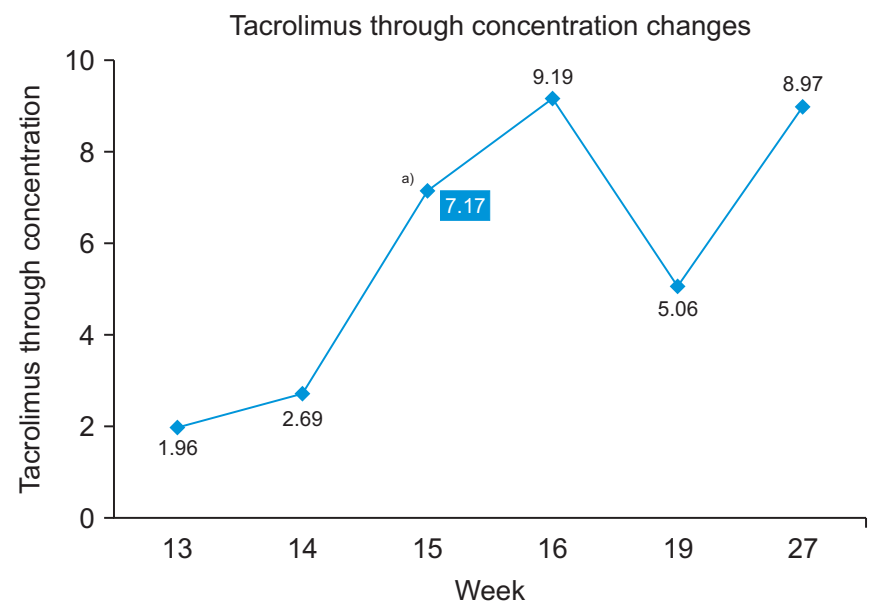

Fig. 3. Changes in tacrolimus trough levels. ${ }^{\text {a) }}$ Ketoconazole $100 \mathrm{mg} \mathrm{bd}$ added.

tacrolimus dose of $4 \mathrm{mg} /$ day. After administering the anti-TB medications, his tacrolimus level became much lower than the target value $(1.96 \mathrm{ng} / \mathrm{mL})$, even after increasing the tacrolimus dose to $10 \mathrm{mg} / \mathrm{day}$. As his tacrolimus level did not reach the target value $(8-10 \mathrm{ng} / \mathrm{mL})$, we added ketoconazole (100 mg/day) (Fig. 3). Frequent monitoring of tacrolimus levels, liver function, and renal function was performed. After adding ketoconazole, his tacrolimus levels reached the target value, his renal function remained

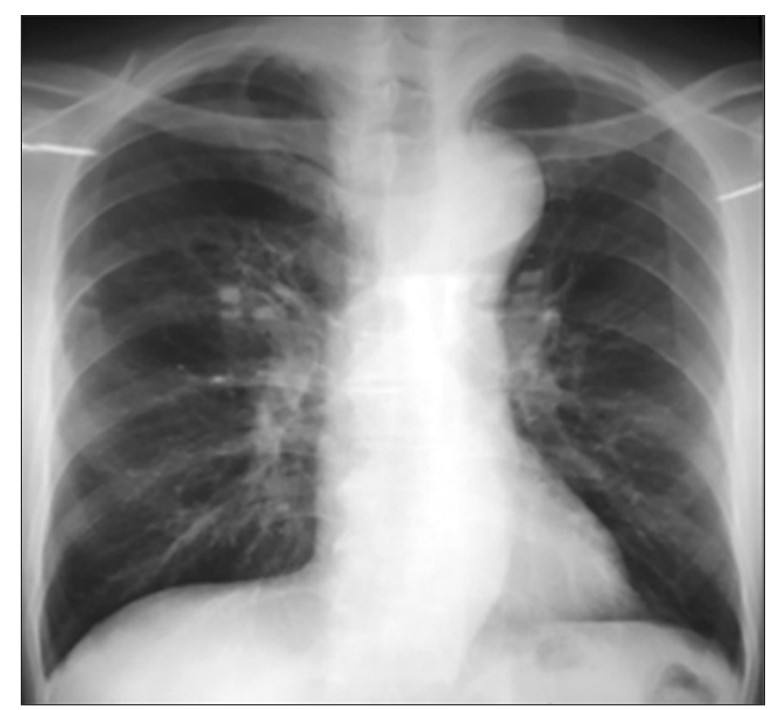

Fig. 4. Recheck chest radiograph (posteroanterior view) taken 6 months after anti-tuberculosis treatment.

normal, and his atrial fibrillation disappeared. Therefore, coadministration of ketoconazole has the benefits of reducing the tacrolimus dose while allowing to reach the target tacrolimus level, and consequently reducing the medical costs. The estimated immunosuppressant cost with ketoconazole was $\$ 120$ per week, and that without ketoconazole was $\$ 225$ per week. 
The patient continued the anti-TB drugs for 6 months (complete course). Glycemic control was performed initially with insulin and later with oral hypoglycemic agents. Hepatotoxicity did not occur with anti-TB medications. His recheck sputum examination showed no AFB, and chest radiography (posteroanterior view) showed considerable improvement (Fig. 4).

\section{DISCUSSION}

Southeast Asia has the highest incidence rate of TB, and the prevalence of active TB is estimated to be $1.2 \%-6.4 \%$ in developed countries and up to $15 \%$ in highly endemic areas [9]. The risk in SOT recipients is estimated to be 20-74 times higher than that in the general population, with a high mortality rate of up to $30 \%$. The mortality rate of TB is higher in patients with disseminated disease, prior rejection, and anti-T-cell antibody therapy.

More than $50 \%$ of renal transplant recipients develop TB within the first year of transplantation [10]. TB mainly occurs during the first year (66.6\%), which could be explained by a possible reactivation of an undetected latent infection. For this reason, TB should be considered as one of the differential diagnoses in patients with fever of unknown origin, especially during the first year after renal transplantation. In the present case, chest radiography and CT suggested the presence of TB, indicating that these two diagnostic methods are complementary rather than exclusive [11].

It is also important to consider that the interactions between anti-TB medications and immunosuppressive drugs, such as rifampicin, can lower the serum levels of calcineurin inhibitors [12] and increase the risk of renal graft rejection. Therefore, it is important to monitor the serum levels of these drugs and make appropriate adjustments. Treating TB in SOT patients is challenging. The drug-todrug interaction between tacrolimus and rifampicin (the cornerstone of TB treatment) poses the risk of rejection of the transplanted organ or TB treatment failure. This interaction leads to decreased tacrolimus levels and may result in an increased risk of rejection. The effect of the drug-todrug interaction between tacrolimus and rifampicin can be managed by increasing the dose of tacrolimus to reach the therapeutic levels.

The diagnosis of TB in transplant recipients is often delayed. In our case, the delay in diagnosis from the onset of symptoms ranged from 15 days to 2 months. Active TB is diagnosed when AFB are observed on smear microscopy and mycobacteria are isolated in the body fluid culture. AFB blood cultures should be performed if disseminated TB is suspected. TB in renal transplant patients manifests with varied and nonspecific symptoms. Pulmonary and disseminated TB are the most common subtypes and require longer use of anti-TB medications. These treatments are associated with high toxicity, high mortality, and appreciable rates of rejection and graft loss. TB should be considered when the patient presents with febrile syndrome of unknown origin, especially during the first year after renal transplantation, and its treatment should be rigorous and strictly monitored.

TB is common in Myanmar. Isoniazid and rifampicin are the most commonly used drugs for TB treatment. Anti-TB regimens containing rifampicin can lower the levels of calcineurin inhibitors. Patients need to be closely monitored for drug interactions with immunosuppressive medications used after SOT, given the increased risk of rejection. Rifampicin is used in the treatment of TB because of its potent $M$. tuberculosis-sterilizing action. Rifampicin is a strong inducer of CYP3A4, leading to increased metabolism of calcineurin inhibitors, mammalian target of rapamycin inhibitors, mycophenolate mofetil, and corticosteroids. Rifabutin is a less potent cytochrome inducer. Drug levels need to be closely monitored at the initiation of TB therapy, after the discontinuation of rifampicin or rifabutin, or with any adjustment of immunosuppressant dosing [13].

Rifabutin is an alternative drug to rifampicin with fewer interactions with tacrolimus [14]. However, it is not easily available in Myanmar. The dose of calcineurin inhibitors should be increased (3-5 times), but this results in higher costs and more adverse effects. Adding ketoconazole to tacrolimus can increase the serum levels of tacrolimus but might reduce the efficacy of anti-TB medications. Clinical adjustments in dose and duration are important in anti-TB treatment [15]. Our patient was free from serious complications such as graft failure and anti-TB drug resistance.

This case suggests that anti-TB drugs reduce the levels of tacrolimus and may cause graft failure if prompt interventions are not provided. Frequent monitoring of tacrolimus levels is necessary in these cases. Drug interactions are an important consideration in the care of patients after renal transplantation. 


\section{ACKNOWLEDGMENTS}

\section{Conflict of Interest}

No potential conflict of interest relevant to this article was reported.

\section{ORCID}

Phyo Wai Lwin

Yi Yi Htun

https://orcid.org/0000-0002-5521-9080

Aung Kyaw Myint https://orcid.org/0000-0001-8854-0164

Htar Kyi Swe

https://orcid.org/0000-0002-1622-934X

\section{Author Contributions}

Conceptualization: all authors. Formal analysis, Data curation, Methodology: PWL, YYH, HKS. Project administration: all authors. Visualization: all authors. Writing-original draft: PWL, YYH, HKS. Writing-review \& editing: all authors.

\section{Additional Contributions}

We thank our colleagues in University of Medicine, Mandalay, Mandalay General Hospital in management and staff for their support. And also thanks for our renal transplant team, laboratory team and participant.

\section{REFERENCES}

1. Flynn JL, Chan J. Immunology of tuberculosis. Annu Rev Immunol 2001;19:93-129.

2. Kaufmann SH, Cole ST, Mizrahi V, Rubin E, Nathan C. Mycobacterium tuberculosis and the host response. $\mathrm{J}$ Exp Med 2005;201:1693-7.

3. Anand M, Nayyar E, Concepcion B, Salani M, Schaefer $\mathrm{H}$. Tuberculosis in kidney transplant recipients: a case series. World J Transplant 2017;7:213-21.

4. Zhang R. Modern immunosuppressive therapy in kidney transplantation. Open J Organ Transpl Surg 2013;3: 22-31.

5. Lemahieu WP, Maes BD, Verbeke K, Vanrenterghem Y. CYP3A4 and P-glycoprotein activity in healthy controls and transplant patients on cyclosporin vs. tacrolimus vs. sirolimus. Am J Transplant 2004;4:1514-22.

6. Scholten EM, Cremers SC, Schoemaker RC, Rowshani AT, van Kan EJ, den Hartigh J, et al. AUC-guided dosing of tacrolimus prevents progressive systemic overexposure in renal transplant recipients. Kidney Int 2005;67:2440-7.

7. el-Dahshan KF, Bakr MA, Donia AF, Badr Ael-S, Sobh MA. Co-administration of ketoconazole to tacrolimus-treated kidney transplant recipients: a prospective randomized study. Nephrol Dial Transplant 2004;19: 1613-7.

8. El-Dahshan KF, Bakr MA, Donia AF, Badr Ael-S, Sobh MA. Ketoconazole-tacrolimus coadministration in kidney transplant recipients: two-year results of a prospective randomized study. Am J Nephrol 2006;26: 293-8.

9. Subramanian AK, Morris Ml; AST Infectious Diseases Community of Practice. Mycobacterium tuberculosis infections in solid organ transplantation. Am J Transplant 2013;13 Suppl 4:68-76.

10. Chen $\mathrm{CH}$, Lian JD, Cheng $\mathrm{CH}$, Wu MJ, Lee WC, Shu $\mathrm{KH}$. Mycobacterium tuberculosis infection following renal transplantation in Taiwan. Transpl Infect Dis 2006;8:148-56.

11. Melchor JL, Gracida C, Ibarra A. Increased frequency of tuberculosis in Mexican renal transplant recipients: a single-center experience. Transplant Proc 2002;34:78-9.

12. Marques ID, Azevedo LS, Pierrotti LC, Caires RA, Sato VA, Carmo LP, et al. Clinical features and outcomes of tuberculosis in kidney transplant recipients in Brazil: a report of the last decade. Clin Transplant 2013;27:E169-76.

13. Walsh R, Ortiz J, Foster P, Palma-Vargas J, Rosenblatt $S$, Wright F. Fungal and mycobacterial infections after Campath (alemtuzumab) induction for renal transplantation. Transpl Infect Dis 2008;10:236-9.

14. Finch CK, Chrisman CR, Baciewicz AM, Self TH. Rifampin and rifabutin drug interactions: an update. Arch Intern Med 2002;162:985-92.

15. Sundaram M, Adhikary SD, John GT, Kekre NS. Tuberculosis in renal transplant recipients. Indian J Urol 2008;24:396-400. 